PROCEEDINGS OF THE

AMERICAN MATHEMATICAL SOCIETY

Volume 124, Number 1, January 1996

\title{
AN INTEGRAL GENERALIZATION OF THE $q$-BINOMIAL THEOREM AND AN APPLICATION
}

\author{
YUNKANG LIU
}

(Communicated by Hal L. Smith)

\begin{abstract}
An integral identity which generalizes the $q$-binomial theorem is presented. This identity is used to determine the exponential expansion of the solution of an integro-differential equation.
\end{abstract}

\section{INTRODUCTION}

One of the most important summation formulas for basic hypergeometric series is given by the $q$-binomial theorem

$$
{ }_{1} \phi_{0}(a ;-; q, z)=\sum_{n=0}^{\infty} \frac{(a ; q)_{n}}{(q ; q)_{n}} z^{n}=\frac{(a z ; q)_{\infty}}{(z ; q)_{\infty}}, \quad|z|<1,|q|<1,
$$

which was derived by Cauchy (1843), Heine (1847) and by other mathematicians (see, e.g., Gasper and Rahman [1]). Motivated by our investigation of the so-called pantograph integro-differential equation [3], we formulate the following integral identity

$$
\begin{aligned}
& 1+\sum_{n=1}^{\infty} \int_{-1}^{1} \ldots \int_{-1}^{1} \frac{\prod_{i=1}^{n}\left(1-a \prod_{j=1}^{i-1} q_{j}\right)}{\prod_{i=1}^{n}\left(1-\prod_{j=1}^{i} q_{j}\right)} d \mu\left(q_{1}\right) \cdots d \mu\left(q_{n}\right) \\
& =\prod_{n=0}^{\infty} \frac{1-a \int_{-1}^{1} q^{n} d \mu(q)}{1-\int_{-1}^{1} q^{n} d \mu(q)},
\end{aligned}
$$

where $a$ is a complex number and $\mu(q)$ a complex function of bounded variation over $[-1,1]$. For simplicity, all integrals considered in this paper are of Riemann-Stieltjes type. To guarantee convergence of the series and products in (2) we assume that $\mu$ satisfies

$$
\int_{-1}^{1}|d \mu(q)|<1
$$

and

$$
\int_{-1}^{1} \frac{1}{1-|q|}|d \mu(q)|<\infty
$$

Received by the editors July 25, 1994.

1991 Mathematics Subject Classification. Primary 33D99; Secondary 45J05.

(C)1996 American Mathematical Society 
It is easy to see that (2) implies (1) by letting $\mu(q)=0$ for $q \in\left[-1, q_{0}\right]$ and $\mu(q)=z$ for $q \in\left[q_{0}, 1\right]$, where $|z|<1,\left|q_{0}\right|<1$.

Denote the left-hand side of $(2)$ by $\Phi(a ; \mu(\cdot))$. One consequence of $(2)$ is the product formula $\Phi(a ; \mu(\cdot)) \Phi(b ; a \mu(\cdot))=\Phi(a b ; \mu(\cdot))$, where $b$ is a complex constant. If we replace $\mu$ by $-a^{-1} \mu$ in (2) and then let $a \rightarrow \infty$ we obtain the integral identity

$$
\begin{aligned}
& 1+\sum_{n=1}^{\infty} \int_{-1}^{1} \ldots \int_{-1}^{1} \frac{\prod_{i=1}^{n} q_{i}^{n-i}}{\prod_{i=1}^{n}\left(1-\prod_{j=1}^{i} q_{j}\right)} d \mu\left(q_{1}\right) \cdots d \mu\left(q_{n}\right) \\
& =\prod_{n=0}^{\infty}\left(1+\int_{-1}^{1} q^{n} d \mu(q)\right)
\end{aligned}
$$

where $\mu$ is a complex function of bounded variation over $[-1,1]$ that obeys (4).

\section{THE PROOF OF (2)}

Let $\mu_{n}(q)=\int_{-1}^{q} p^{n} d \mu(p), n \geq 0$, be such that $d \mu_{n}(q)=q^{n} d \mu(q)$. It is straightforward to verify formally for any constant $n \geq 0$ the identity

$$
\left(1-\mu_{n}(1)\right) \Phi\left(a ; \mu_{n}(\cdot)\right)=\left(1-a \mu_{n}(1)\right) \Phi\left(a ; \mu_{n+1}(\cdot)\right) .
$$

From the preceding identity we derive by induction that

$$
\left[\prod_{n=0}^{N}\left(1-\mu_{n}(1)\right)\right] \Phi(a ; \mu(\cdot))=\left[\prod_{n=0}^{N}\left(1-a \mu_{n}(1)\right)\right] \Phi\left(a ; \mu_{N+1}(\cdot)\right)
$$

holds for any integer $N \geq 0$. Noting that $\lim _{N \rightarrow \infty} \Phi\left(a ; \mu_{N+1}(\cdot)\right)=1$, we obtain (2) by letting $N \rightarrow \infty$ in the preceding identity. The preceding argument can be made rigorous by exploiting our assumptions (3) and (4). For instance, the series on the left-hand side of (2) converges absolutely if

(6)

$$
\limsup _{n \rightarrow \infty}\left\{\int_{-1}^{1} \ldots \int_{-1}^{1} \frac{\prod_{i=1}^{n}\left(1+|a| \prod_{j=1}^{i-1}\left|q_{j}\right|\right)}{\prod_{i=1}^{n}\left(1-\prod_{j=1}^{i}\left|q_{j}\right|\right)}\left|d \mu\left(q_{1}\right)\right| \cdots\left|d \mu\left(q_{n}\right)\right|\right\}^{1 / n}<1 .
$$

To see that (3) and (4) imply (6), we choose a constant $p \in(0,1)$ such that

$$
\int_{p}^{1} \frac{1+|a|}{1-q}|d \mu(q)|+\int_{-1}^{-p} \frac{1+|a|}{1+q}|d \mu(q)| \leq \int_{-1}^{1}|d \mu(q)| .
$$

By separating the integral domain $[-1,1]^{n}$ into $[-p, p]^{n}$ and $[-1,1]^{n}-[-p, p]^{n}$, we obtain

$$
\begin{aligned}
& \int_{-1}^{1} \ldots \int_{-1}^{1} \frac{\prod_{i=1}^{n}\left(1+|a| \prod_{j=1}^{i-1}\left|q_{j}\right|\right)}{\prod_{i=1}^{n}\left(1-\prod_{j=1}^{i}\left|q_{j}\right|\right)}\left|d \mu\left(q_{1}\right)\right| \cdots\left|d \mu\left(q_{n}\right)\right| \\
\leq & \frac{(-|a| ; p)_{n}}{(p ; p)_{n}}\left(\int_{-p}^{p}|d \mu(q)|\right)^{n}+\left(\int_{p}^{1} \frac{1+|a|}{1-q}|d \mu(q)|+\int_{-1}^{-p} \frac{1+|a|}{1+q}|d \mu(q)|\right)^{n} \\
\leq & \left(\frac{(-|a| ; p)_{\infty}}{(p ; p)_{\infty}}+1\right)\left(\int_{-1}^{1}|d \mu(q)|\right)^{n}
\end{aligned}
$$

which implies (6). 


\section{An Application of (2) AND (5)}

Consider the pantograph integro-differential equation

(7)

$$
y^{\prime}(t)=a y(t)+b \int_{0}^{1} y(q t) d \mu(q)+c \int_{0}^{1} y^{\prime}(q t) d \mu(q), \quad t>0, \quad y(0)=1,
$$

where $a, b$ and $c$ are complex constants, $a \neq 0$, and $\mu$ is a complex function of bounded variation over $[0,1]$ that obeys

1. $c \int_{0}^{1} q^{n} d \mu(q) \neq 1$, for all $n=0,1, \ldots$;

2. $|b| \int_{0}^{1}|d \mu(q)|<|a|, \int_{0}^{1} \frac{1}{1-q}|d \mu(q)|<\infty$;

3. $\int_{0}^{1} q^{n}|d \mu(q)| \rightarrow 0$ as $n \rightarrow \infty$.

Under the last assumption it is proved in [3] that the first one is both necessary and sufficient for the existence and uniqueness of a smooth solution of (7).

In order to study the asymptotic behaviour of the solution of (7), we seek a solution of the form

(8)

$$
\begin{aligned}
y(t)=\alpha\left\{e^{a t}+\right. & \sum_{n=1}^{\infty}(-a)^{-n} \int_{0}^{1} \ldots \\
& \left.\int_{0}^{1} \frac{\prod_{i=1}^{n}\left(b+a c \prod_{j=1}^{i-1} q_{j}\right)}{\prod_{i=1}^{n}\left(1-\prod_{j=1}^{i} q_{j}\right)} e^{q_{1} \cdots q_{n} a t} d \mu\left(q_{1}\right) \cdots d \mu\left(q_{n}\right)\right\},
\end{aligned}
$$

where $\alpha$ is a constant to be determined. It is trivial to verify that the preceding series satisfies (7) if and only if $\alpha$ obeys

$$
\alpha\left\{1+\sum_{n=1}^{\infty}(-a)^{-n} \int_{0}^{1} \cdots \int_{0}^{1} \frac{\prod_{i=1}^{n}\left(b+a c \prod_{j=1}^{i-1} q_{j}\right)}{\prod_{i=1}^{n}\left(1-\prod_{j=1}^{i} q_{j}\right)} d \mu\left(q_{1}\right) \cdots d \mu\left(q_{n}\right)\right\}=1
$$

which, by (2) if $b \neq 0$ or by (5) if $b=0$, implies that

$$
\alpha=\prod_{n=0}^{\infty} \frac{1+a^{-1} b \int_{0}^{1} q^{n} d \mu(q)}{1-c \int_{0}^{1} q^{n} d \mu(q)} .
$$

One obvious consequence of (8) and (9) is that when $\Re a>0$ the solution $y(t)$ of (7) increases (in modulus) exponentially. More specifically,

$$
\lim _{t \rightarrow \infty} y(t) e^{-a t}=\prod_{n=0}^{\infty} \frac{1+a^{-1} b \int_{0}^{1} q^{n} d \mu(q)}{1-c \int_{0}^{1} q^{n} d \mu(q)} \neq 0,
$$

which partly proved a conjecture posed in [3]. Another consequence is that when $\Re a=0$ the solution $y(t)$ of $(7)$ is uniformly bounded on $[0, \infty)$. We can also use (8) to discuss the asymptotic behaviour of the solution in the case of $\Re a<0$. However, this case has already been studied in [3] under a slightly weaker assumption and for the more general equation

$$
y^{\prime}(t)=a y(t)+\int_{0}^{1} y(q t) d \mu_{1}(q)+\int_{0}^{1} y^{\prime}(q t) d \mu_{2}(q), \quad t>0, \quad y(0)=1,
$$


where $\mu_{1}$ and $\mu_{2}$ are complex functions of bounded variation over $[0,1]$.

Remark. An exponential expansion that is much more complicated than (8) does exist for the solution of (10). However, the corresponding integral identity is less elegant and the proof is too lengthy to be presented in this article.

\section{ACKNOWLEDGMENTS}

The author wishes to thank his supervisor Dr. A. Iserles for many stimulating discussions on this subject. The author is supported by scholarships from Cambridge Overseas Trust, CVCP, Fitzwilliam College and C.T. Taylor Fund.

\section{REFERENCES}

1. G. Gasper and M. Rahman, Basic hypergeometric series, Cambridge Univ. Press, Cambridge, 1990. MR 91d:33034

2. A. Iserles, On the generalized pantograph functional-differential equation, European J. Appl. Math. 4 (1993), 1-38. MR 94f:34127

3. A. Iserles and Y. Liu, On pantograph integro-differential equations, J. Integral Equations Appl. 6 (1994), 213-237. MR 95g:45008

Department of Applied Mathematics and Theoretical Physics, University of CamBridge, Silver Street, Cambridge, CB3 9EW, England

E-mail address: yl@amtp.cam.ac.uk 